\title{
La vivienda mínima: una revisión del desarrollo del concepto en Colombia
}

\author{
Minimum housing: a review of the development of the concept in Colombia \\ Moradia mínima: uma revisão do desenvolvimento do conceito na Colômbia
}

\author{
Jorge Correa Orozco. M.Sc. en Hábitat. \\ jorge.correao@cecar.edu.co \\ (iD) https://orcid.org/0000-0003-1569-9304 \\ Corporación Universitaria del Caribe-CECAR. Colombia
}

Recibido: Agosto 15 de 2017

Aceptado: Julio 23 de 2018

Publicado: Diciembre de 2018

\section{RESUMEN}

El presente artículo hace la revisión del concepto de vivienda mínima en Bogotá. Para ello, toma como punto de partida las ideas del movimiento moderno, complementado esto con la revisión de la normatividad colombiana. El objetivo es poder evidenciar, a la luz de la normativa, el decrecimiento paulatino que sufrió la vivienda de interés social (VIS) con cada decisión que se tomaba, dejando ver que el panorama actual sobre el tema es complejo y no muy alentador. La VIS, más que un tema de resolución técnica, necesita ser repensada desde las políticas públicas y desde la normativa.

Palabras clave: Vivienda mínima; movimiento moderno; normatividad; políticas públicas.

\begin{abstract}
This article reviews the concept of minimum housing in Bogotá. To do this, it takes, as a starting point, the ideas of the modern movement, complemented by the revision of the Colombian normativity. The objective is to be able to show, in light of the regulations, the gradual decrease in social interest housing (VIS) with each decision that was made, showing that the current panorama on the subject is complex and not very encouraging. The VIS, more than a technical resolution issue, needs to be rethought from public policies and from regulations.
\end{abstract}

Keywords: Minimum housing; modern movement; regulations; public policies.

\section{RESUMO}

Esta pesquisa analisa o conceito de habitação mínima. Para fazer isso, começa no início do conceito: o movimento moderno na Europa. Bem como o contexto em que o conceito e finalidade que nasceu. Isso complementa isso com a revisão dos regulamentos de Bogotá sobre habitação voltada para setores de baixa renda, as particularidades de cada período e as ideias subjacentes aos padrões de design. O objetivo é descrever o impacto negativo das decisões em política nos padrões de habitação social no século XX em Bogotá. Esses impactos negativos nos permitem ver a dificuldade das políticas públicas para atingir seus objetivos.

Palavras chave: Habitação mínima; movimento moderno; regulamentos; políticas públicas.

Como citar (APA)

Correa Orozco, J. (2018). La vivienda mínima: una revisión del desarrollo del concepto en Colombia. Procesos Urbanos 5:34-41. DOI: https://doi.or$\mathrm{g} / 10.21892 / 2422085 \mathrm{X} .406$ 


\section{Introducción}

El primer propósito de este artículo consiste en revisar el concepto de vivienda mínima, con el fin de precisar el origen y su adopción en el contexto nacional. El concepto de vivienda mínima tiene su origen en el movimiento moderno, en la Europa de posguerra. Esta vivienda se empieza a gestar con el fin de proveerla a la población de menos recursos, para lo cual se crean prototipos que traen consigo toda una estructura de ciudad y un modo de habitar diferente.

Estos prototipos son adoptados en Latinoamérica, y Colombia no es la excepción. A raíz de las migraciones sufridas y la creciente población obrera, se hace necesario adoptar medidas parecidas a las europeas para proveer de vivienda a estas poblaciones. A raíz de esto, se adaptan a nuestro entorno las soluciones planteadas por los europeos, con lo cual se empieza una serie de etapas de producción de vivienda. Estas etapas estuvieron marcadas por cambios sociales y políticos, y repercutieron en forma directa en las formas de habitar de la población colombiana.

El segundo propósito de este artículo es revisar tales etapas a la luz de las decisiones político-económicas y las normas que rigieron la vivienda, con el fin de precisar qué cambios y qué tipo de vivienda se le ofreció a la población en cada una de ellas. La investigación identifica cinco etapas o fases al respecto. Tal distinción se hizo tomando como base la calidad de la vivienda, es decir, lo ofrecido al habitante en términos de habitabilidad y calidad arquitectónica.

\section{El nacimiento del concepto en el movimiento moderno}

Los arquitectos reunidos en los Congresos Internacionales de Arquitectura Moderna-CIAM, abordaron el tema de la vivienda mínima. En el II Congreso, celebrado en Frankfurt en 1929, se tomó como eje central "La vivienda para el mínimo nivel de vida"; en este, el arquitecto Walter Gropius contribuyó con un texto titulado "Los fundamentos sociológicos de la vivienda mínima para la población obrera de la ciudad", en el que se leen, entre otros, los siguientes argumentos de Gropius (citado en Aymonino, 1973):

\begin{abstract}
"La clarificación de los datos históricos de la sociedad debe seguir adelante para que se pueda encontrar el tamaño óptimo de la vivienda que satisfaga las necesidades de la vida al menor precio posible, ya que, a consecuencia del cambio de los condicionantes, el problema de la vivienda no puede ser resuelto con la mera reducción del tamaño de las habitaciones y de superficie útil de la usual vivienda de mayor tamaño. El nuevo problema debe enfocarse mediante el conocimiento de las exigencias naturales y sociales mínimas (...) La clave de la cuestión del mínimo nivel de vida está en saber el elemento mínimo de espacio, aire, luz, calor, que el hombre necesita para desarrollar totalmente sus funciones vitales mediante un alojamiento, es decir, el minimun vivendi en lugar de un modus non moriendi." (p. 120).
\end{abstract}

Estos argumentos intentan soportar el concepto de vivienda mínima desde los análisis higienistas y sociológicos. Entre los primeros, se refuerza la idea de hacer viviendas ventiladas con el fin de expulsar el aire viciado e introducir aire puro, renovándolo constantemente; espacios iluminados a través de ventanas grandes para aprovechar la luz del sol y producir calor. Plantear viviendas con habitaciones independientes: una habitación por cada adulto con el fin de preservar la intimidad y separar las actividades de los adultos de las de los menores. Además, se buscó el mejoramiento de elementos vitales, como el agua y la evacuación constante de residuos.

Por otro lado, el análisis sociológico identifica que las estructuras de la sociedad son cambiantes, la composición de la familia (el número y de roles de sus miembros) es diferente, el papel de la mujer ha cambiado, lo cual deja ver que existen necesidades diferentes a las de la antigua forma familiar. Por ello, la vivienda debe cambiar y responder a estas nuevas formas de unión familiar. Gropius, (citado por Aymonino, 1973) argumenta que "la forma organizativa de las tareas del hogar para mujeres y hombres solteros, para niños y adultos, viudos o casados, para jóvenes matrimonios o para sociedades e ideologías de diferente estructura, está ligada inseparablemente al problema de la vivienda" (p. 120). Así, las necesidades varían con los cambios en los grupos poblacionales: la vivienda dirigida a dichos grupos debe contener elementos específicos que respondan a sus necesidades y debe ayudar a erradicar las enfermedades que aquejan a la población de esa época.

De este congreso salió como resultado un prototipo de vivienda mínima que sería adoptado y adaptado en muchos lugares incluyendo Colombia, el Existenzminimun (ver Figura 1). En palabras de Saldarriaga:

"Este modelo resumió algunos de los avances alcanzados en diversos proyectos realizados en Holanda y Alemania a lo largo de esa década [1920]. El modelo es muy sencillo y corresponde cabalmente a los principios del funcionalismo y de la racionalización constructiva. Este modelo fue adaptado posteriormente en otros lugares y tuvo una difusión prácticamente universal." (1996, p .20).

La vivienda mínima atendió los requerimientos de la higiene por lo cual se convirtió en una máquina de habitar' (Le Corbusier, citado en CIAM, 1954²), es decir, un elemento puramente funcional en donde no existe otra diferencia que la reducción de espacio, la esquematización y simplificación de la vida de quienes allí habitan. Las diferencias culturales y la diferencia en los comportamientos de los habitantes tuvieron, al fin de cuentas, una prioridad menor, una respuesta menor del diseño de la vivienda mínima. Así, la filosofía de esta se constituye en:

"(...) la de la "vivienda higiénica", esta concepción trae de la mano la puesta en funcionamiento de un sistema de provisión de vivienda y de hábitat donde "el nuevo ser social está dispuesto a aceptar una nueva forma de vivienda austera, esquemática, eficiente y funcional. La diferencia entre una gran casa, un apartamento o una vivienda mínima no se planteó en lo conceptual sino en lo cuantitativo. Todas deberían ser máquinas de habitar, pero de diferente tamaño." (Saldarriaga, 1996, p. 20).

La vivienda para el mínimo modo de vida tomaría auge como solución a los problemas habitacionales de las clases con menos recursos en la Europa de posguerra. Si bien es cierto que el concepto de vivienda mínima tuvo sus bases en la sociología y en los planteamientos higienistas, su fin último fue el de cumplir con lo dispuesto por los partidarios de la higiene, dejando de lado los valiosos aportes que intentaba explicar Walter Gropius a través de sus argumentos sobre los cambios en la estructura social de los pueblos y su repercusión en el diseño de la vivienda, y sobre la forma de cubrir las nuevas necesidades que podían surgir y desechar las que en algún sentido eran obsoletas.

La definición de este nuevo tipo de vivienda estuvo marcada por un sentido físico que contempló los elementos naturales 


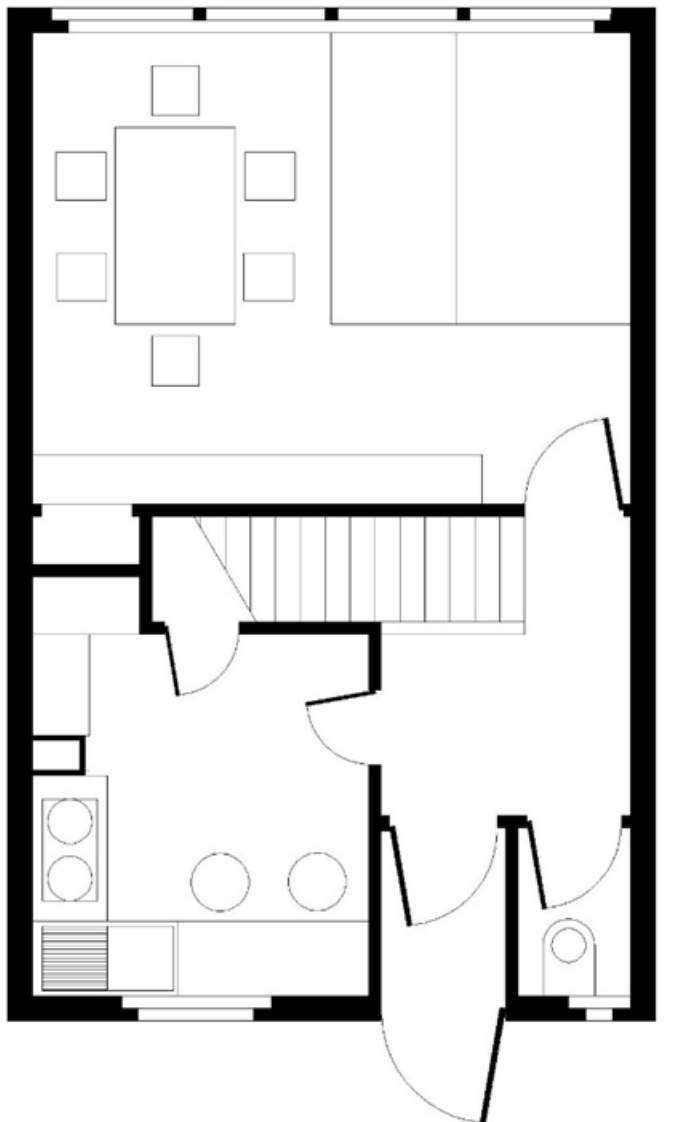

Figura 1. El Existenzminimun. CIAM, 1929 Fuente: Elaboración propia

del problema y relegó de alguna forma los sociales, enfatizando en la funcionalidad y esquematización de la vida de quienes allí habrían de habitar. Es por esto que, en términos cualitativos, el concepto de la vivienda mínima no se definió completamente.

\section{La vivienda mínima en Colombia}

En Latinoamérica, la vivienda aparece en respuesta a "la masividad, pobreza extrema, urbanización, desborde de los mecanismos formales de organización de la ciudad, segregación social y espacial, coincidente con la posguerra europea." (Pelli, 2006, p. 94) Colombia empieza a sufrir cambios análogos en las estructuras sociales y en las ciudades debido al fenómeno de la violencia. De esta forma, toman fuerza las premisas del movimiento moderno, empezando en Colombia la construcción de viviendas higiénicas dirigidas a los sectores obreros y campesinos. Para esto, se establecen normas mínimas de vivienda.

Normas que cambiarán a lo largo del siglo XX, debido a diversos factores políticos y económicos. Los cambios pueden resumirse en cinco fases, teniendo como punto de partida la revisión de autores como Samuel Jaramillo, Alberto Saldarriaga y Gilberto Arango. A través de ellas, se puede ver claramente la disminución gradual del área de la vivienda destinada a los estratos bajos de la población. Estas fases son las siguientes.

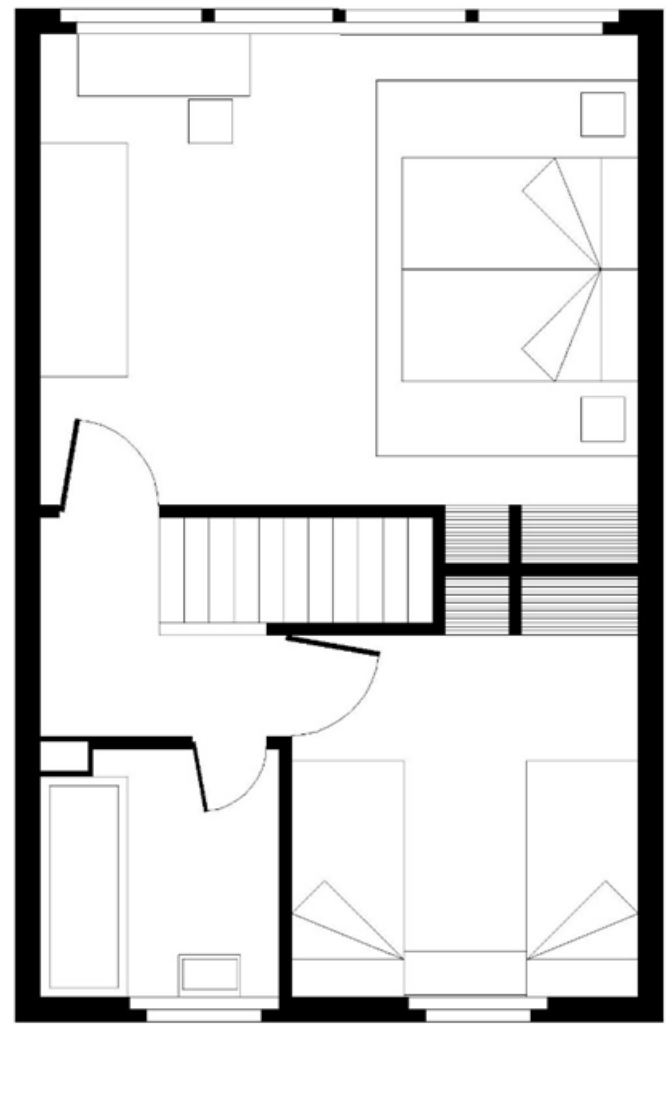

La influencia del movimiento moderno en la fase de las viviendas higiénicas

El primer periodo, comprendido entre 1918 hasta 1950, se enmarca a partir de la promulgación de la Ley 46 de 1918, la cual otorgó recursos para la construcción de viviendas higiénicas para la clase proletaria. Nos recuerda Alberto Saldarriaga que:

"A lo largo del siglo XX, la participación municipal y distrital en la construcción de vivienda para obreros en Bogotá se lleva a cabo a través de la gestión de tres entidades: la Junta de Habitaciones para Obreros, que intervino entre 1919 y 1932; el Instituto de Acción Social, que operó entre 1932 y 1942; y la Caja de Vivienda Popular, que inicia labores a partir de 1942. La Junta de Habitaciones para Obreros se constituye gracias a un acuerdo establecido entre la nación y el municipio, para dar cumplimiento a lo establecido en la Ley 46 de 1918. Es esta la primera entidad de carácter público que tiene a su cargo la financiación de proyectos de vivienda económica en la capital del país." (1996, p. 204).

Dentro de este periodo, se fundó el Banco Central Hipotecario (Ley 170 de 1936), destinado a la captación de ahorro y a la financiación de viviendas. En 1939 se creó el Instituto de Crédito Territorial, orientado inicialmente al fomento de vivienda rural y que, posteriormente, atendería la demanda de vivienda urbana ${ }^{3}$, con la Ley 53 de 1942, la cual amplió la operatividad del ICT y lo autorizó a otorgar préstamos a cooperativas de vivienda, dirigidas a urbanizar y construir viviendas para empleados, profesionales y pequeños comerciantes en las áreas urbanas. 
En este periodo, son importantes el barrio Muzú - debido a que sigue la tradición moderna en la solución de vivienda mínima para la clase obrera y se proyecta bajo los parámetros concebidos en la Carta de Atenas- y el prototipo de vivienda mínima nacido en el CIAM de 1929: El Existenzminimun. Al respecto, Alberto Saldarriaga anota:

"En el plan urbanístico del barrio, se aplicaron rigurosamente los principios de urbanismo moderno: una red vial 'incompleta' en la que una sola vía atraviesa el terreno y remata en cul de sac, la desaparición completa de la manzana tradicional y su reemplazo por grupos de predios dispuestos en medio de áreas verdes, una red de vías peatonal como principal enlace entre las viviendas y un centro de servicios deportivos y comunitarios. La vivienda se compone de dos muros paralelos, separados por 5,50 metros. Definen los costados del rectángulo de la casa, el que a su vez se divide en dos secciones transversales, tanto en el primer piso como en el segundo." (1996, p. 134).

Con este primer ejemplo, se indica una nueva forma de vivienda y manera de habitar traídas por la arquitectura moderna y la filosofía de la vivienda mínima. El barrio Muzú constituye la entrada de la modernidad en la vivienda, en la solución de vivienda para la clase obrera y trabajadora.

De esta forma, la vivienda en Colombia toma dos características establecidas en el movimiento moderno. El primer aspecto, es su desarrollo en masa como estrategia para proveer de viviendas a la población campesina que migra constantemente a las principales ciudades. El segundo aspecto, es la adopción de la filosofía de la vivienda mínima como la vivienda higiénica. A raíz de esto, la vivienda colombiana se configura de igual forma que las viviendas europeas: los espacios son completamente idénticos, pero los problemas son diferentes. Desde este punto, la vivienda mínima sufre una ruptura, se adapta al contexto en términos cuantitativos, pero no cualitativos, es decir, se atienden solo los problemas higiénicos y de provisión de vivienda que resultan ser solo la mitad del fenómeno que empezaba a vivir la población colombiana, y se deja de lado, al igual que en los CIAM, los postulados de Gropius, las transformaciones sociales de los pueblos a los cuales esta vivienda se dirige.

\section{Fase de calidad habitacional bajo el estado asistencialista}

En esta segunda fase, se encuentra el Estado como protagonista e inductor del proceso de la edificación en Colombia, fue una época en la que hubo grandes transformaciones demográficas, por el crecimiento de la población urbana y el auge de las migraciones campo-ciudad ligados al proceso de industrialización de las principales ciudades, lo que a su vez agudizó la demanda de vivienda, fundamentalmente en las principales ciudades del país.

A raíz de esto, el Estado planteó mecanismos de intervención frente al problema del déficit habitacional que demandaban las grandes ciudades, que se canalizaron a través de las instituciones del Estado -como el Banco Central Hipotecarioel cual se creó como un sistema de financiación especializado en la rama de la construcción, cuya función era captar ahorro del público y ponerlo, en primer lugar, a disposición de los constructores, y, en segundo lugar, a los usuarios finales, pero a plazos más largos.

El Instituto de Crédito Territorial actuó como promotor estatal encargado de producir y promover vivienda para los sectores de bajos recursos, con subsidio, y también para los estratos medios y altos, pero sin subsidio. Trabajó con recursos del presupuesto nacional, provenientes de su cartera o de empréstitos internacionales, y recibió una fuerte inversión en la década de los años sesenta del programa Alianza para el Progreso ${ }^{4}$, para que emprendiera grandes acciones.

En este contexto, surge el Decreto 51 de 1963, a través del cual se adopta el Plan de Zonificación de la ciudad de Bogotá, para hacer una categorización de la vivienda discriminada por zonas de densidad baja, media y alta; en ella se encuentran las viviendas para los obreros, clasificadas en viviendas $R-6, R-7$, R-8 y R-E.

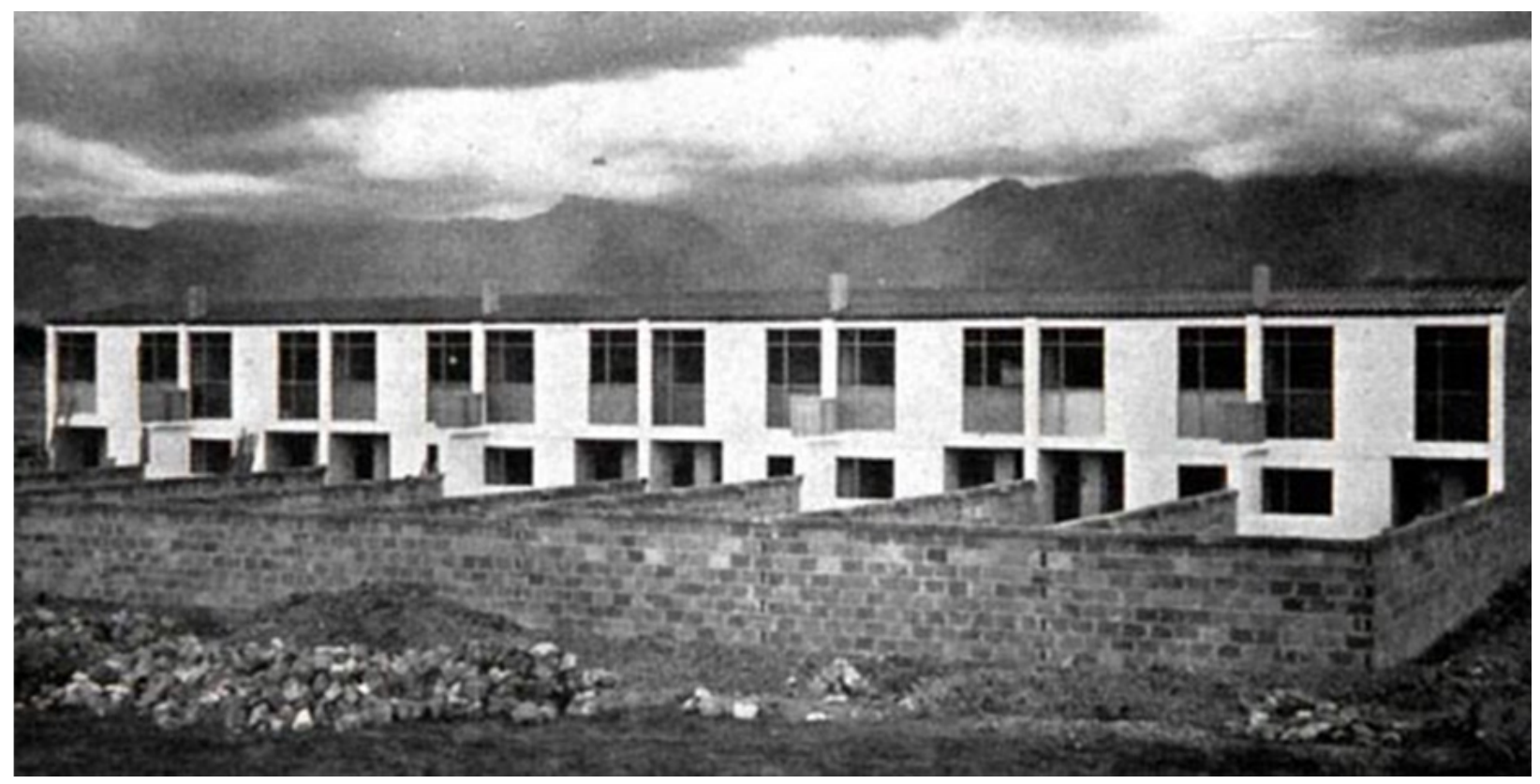

Figura 2. Viviendas terminadas en el barrio Muzú, Bogotá

Fuente: Mendoza Neira (1960) 
Las áreas mínimas de los lotes para cada clasificación son las siguientes: para R-6 se destinaría un lote mínimo de 120 m2; para R-7 es de $90 \mathrm{~m} 2$; para R-8 es de $65 \mathrm{~m} 2$ y para R-E de $65 \mathrm{~m} 2$. Además, se planteó un frente mínimo de 6 metros; se exigían aislamientos laterales de $3 \mathrm{~m}$ para las viviendas aisladas, pareadas o gemelas; y un aislamiento posterior de $3 \mathrm{~m}$ como mínimo, a partir del segundo piso. Se dispuso una altura de 1 a 3 pisos para viviendas unifamiliares y 2 o 3 pisos para multifamiliares. Estas últimas tendrían un mínimo de dos pisos y un máximo equivalente a dos veces el ancho de la vía. Además, se debía proveer un garaje por cada cuatro viviendas, con las dimensiones de $6 \times 2,85 \mathrm{~m}$. En este periodo, se puede citar como ejemplo las viviendas unifamiliares de la urbanización Timiza, donde hay una exploración urbana y arquitectónica en la solución de la vivienda. Alberto Saldarriaga lo explica así:

"El plan urbanístico de Timiza se basa en el manejo de súper manzanas y de la red peatonal. Está trazado siguiendo una geometría irregular, basada en diagonales que responden a planteamientos paisajísticos y que se relacionan en el conjunto con un parque y un lago." (1996, p. 136).

Es de especial importancia la arquitectura de las casas unifamiliares. En ellas hay una búsqueda formal de la vivienda social que se ha presentado pocas veces en este tipo de proyectos. Saldarriaga anota que:

"La casa se encierra por un muro alto y se desarrolla en dos rectángulos desplazados que generan dos patios diagonales, uno de acceso y otro de servicio. La primera etapa consta de un espacio social y servicios en el primer piso y dos habitaciones en el segundo. Las ampliaciones sucesivas pueden conducir a una vivienda de cuatro alcobas." (1996, p. 136).

Con este ejemplo, se muestra claramente que en ese momento existían las condiciones políticas y económicas para producir vivienda social con calidad arquitectónica, técnica y con una considerable cantidad de espacio que reflejaba la implementación de estándares mínimos más acordes con las condiciones sociales de los usuarios, tal como los plantea la clasificación de vivienda de 1963.

En esta fase, la vivienda social está en manos del Estado que asiste a los grupos sociales más débiles, crea instituciones, gestiona los recursos y plantea las políticas necesarias a través de las cuales proveer de vivienda a estos sectores. De esta forma, el Estado regula los estándares de habitabilidad y las dimensiones mínimas de la vivienda, lejos de las tranzas del mercado, gozando de características espaciales y calidad arquitectónica.

Fase de debilitamiento en la calidad habitacional en el periodo de transición. Acuerdo 7 de 1980

A partir de los años setenta, empieza un proceso de privatización que da paso a las Corporaciones de Ahorro y Vivienda. En ese sentido, la política gubernamental, frente al sector de la edificación y de la producción de vivienda, sufre un gran cambio por el agotamiento del modelo económico adoptado, basado en la sustitución de importaciones industriales, con el cual se pensó en incentivar a los sectores líderes de la economía del país y, en tal sentido, se pretendió fortalecer la edificación como motor del desarrollo económico del mismo Esto trajo consigo el desmonte paulatino del $\mathrm{BCH}$.

A finales de 1972, el presidente Misael Pastrana introdujo el mecanismo de indexación, en el cual los depósitos de los ahorradores y las obligaciones de los deudores se reajustarían al ritmo de la inflación. A lo que se llamó la unidad reajustable, o Unidad de Poder Adquisitivo Constante-UPAC. Además, reemplazó el $\mathrm{BCH}$ (organismo estatal que era el eje central del sistema) por organismos privados (corporaciones de ahorro y vivienda-CAV) que captarían los ahorros y asignarían los préstamos. Así, todos los grupos financieros crearon sus CAV. Se afianzó el sistema UPAC como mecanismo privilegiado de

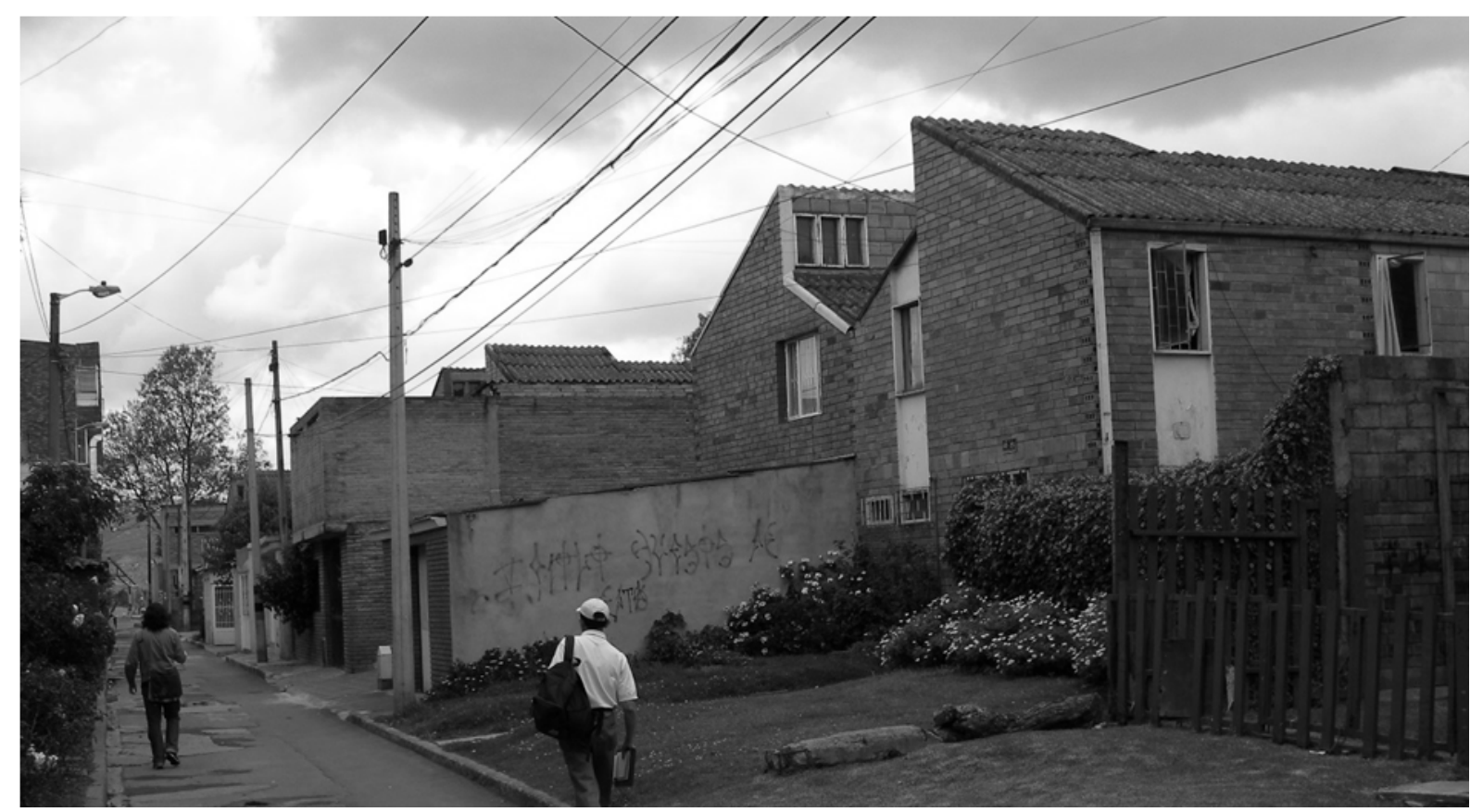

Figura 3. Viviendas terminadas en el barrio Muzú, Bogotá Fuente: Foto del autor (2005) 
captación de ahorro y de financiación de la construcción, el cual se convirtió en el instrumento más importante de movimiento de ahorros voluntarios del país, y contribuyó a la modernización de las prácticas financieras. Así, las corporaciones de ahorro y vivienda alcanzaron un nivel preponderante en el conjunto del sistema financiero de vivienda institucional y desplazaron a las instituciones del Estado en el papel de constructoras directas de vivienda económica.

Con este panorama político se puede referenciar el Acuerdo 7 de 1980. En esta norma se habla de densidad residencial y refiriere al resultado obtenido de aplicar tres requisitos importantes: el índice de habitabilidad, el equipamiento comunal y la volumetría permitida. En el primero de estos, el índice de habitabilidad, el área mínima de la vivienda, se calcula teniendo como referente principal el equivalente a $20 \mathrm{~m} 2$ por alcoba, incluidos en ella las áreas comunes como circulación, escaleras, ascensores, depósitos y administración. De esta forma las viviendas quedaban con las siguientes áreas mínimas:

- Vivienda de una (1) alcoba: área mínima veinte (20 m2).

- Vivienda de dos (2) alcobas: área mínima cuarenta (40 m2).

- Vivienda de tres (3) alcobas: área mínima sesenta (60 m2).

- Vivienda de cuatro (4) alcobas: área mínima ochenta (80 m2) y así sucesivamente.

Como ejemplo de este tipo de vivienda se encuentra Candelaria La Nueva, proyecto realizado por la Caja de Vivienda Popular que se finaliza en 1988 y se encuentra ubicado al suroriente de la ciudad. Consta de viviendas unifamiliares y multifamiliares. Las viviendas unifamiliares se construyen en un lote de $6 \mathrm{~m}$ de frente por $12 \mathrm{~m}$ de fondo. (ver figura 4). Estas viviendas cuadruplican el área actual de una vivienda de interés social, son aún mínimos admisibles en términos cuantitativos y superan con creces los mínimos que rigen actualmente. El mercantilismo de la vivienda es incipiente pero potencial en este periodo.

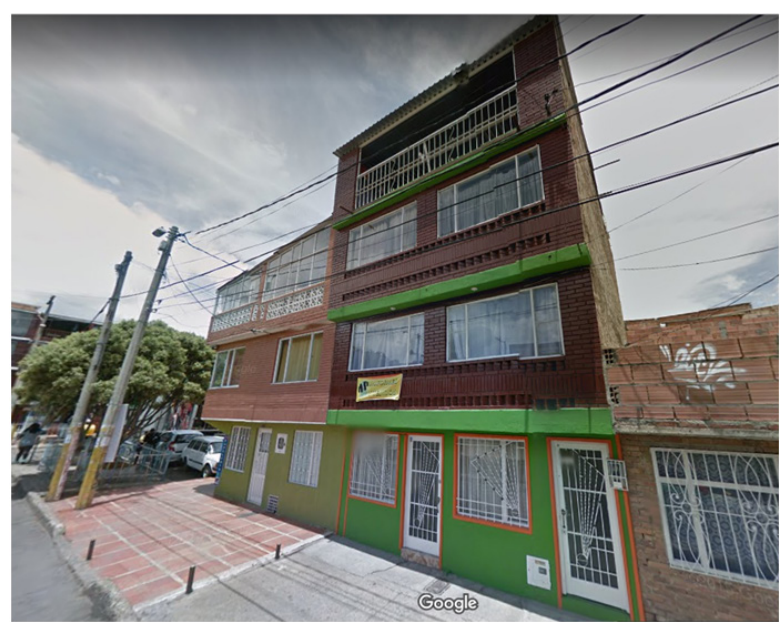

Figura 4. Viviendas modificadas en Candelaria La Nueva, Bogotá. Fuente: Google Street View. 2017

Fase de baja calidad habitacional bajo el periodo de mercado. Acuerdo 734 de 1993.

Este periodo se enmarca por la creación de la Ley 9 de 1989, la cual fue un instrumento que serviría en la consecución de recursos financieros para el desarrollo territorial, instituyendo los usos del suelo, además de establecer los bancos de tierra con el fin de financiar lo establecido en el Artículo 10 de la misma, que involucra todos los aspectos del desarrollo urbano. Con esta ley se establece la política base en cuanto al concepto de vivienda de interés social, la que califica teniendo en cuenta su precio.

Igualmente, la Ley 3 de 1991 creó el Sistema Nacional de Vivienda de Interés Social-Sinavis, el cual contempla actores para el fomento, la ejecución, la asistencia técnica y la financiación, y transformó las condiciones de la acción estatal para la vivienda de interés social, con lo que se comenzó una nueva fase que contó con entidades públicas y privadas que cumplen funciones conducentes a la financiación, construcción, mejoramiento, reubicación, habilitación y legalización de títulos de vivienda.

El instrumento fundamental de la producción de vivienda social del Estado, el ICT, entró en crisis por las siguientes razones: la capacidad operativa se desbordó por las elevadas y espontáneas exigencias a las que se le sometió; el régimen de propiedad territorial fue muy desfavorable, dada su función social, esto le impedía adquirir buenos terrenos y frenaba el desarrollo de proyectos de gran envergadura. Por esta razón, se vio obligado a aceptar cualquier tipo de proyecto de promoción conjunta, así la negociación fuera lesiva o el producto final fuera inconveniente.

La baja calidad de sus viviendas y los sobreprecios ocasionaron que muchos adjudicatarios no pudieran cancelar los créditos y/o se negaran a hacerlo. El sector privado empezó a ingresar al negocio de la construcción de vivienda para grupos populares, lo cual incidió en el mercado del suelo; compró grandes terrenos en áreas de expansión popular, en una proporción mayor a la intención de construir dichos terrenos, lo que generó una gran reserva de los mismos, razón por la cual la tierra urbanizable para construcción de vivienda popular se encareció. En cuanto a la producción estatal, no se hizo nada para transformar el régimen de propiedad territorial que tanto afectó al ICT; tampoco se establecieron mecanismos para favorecer a esta entidad frente a los contratistas.

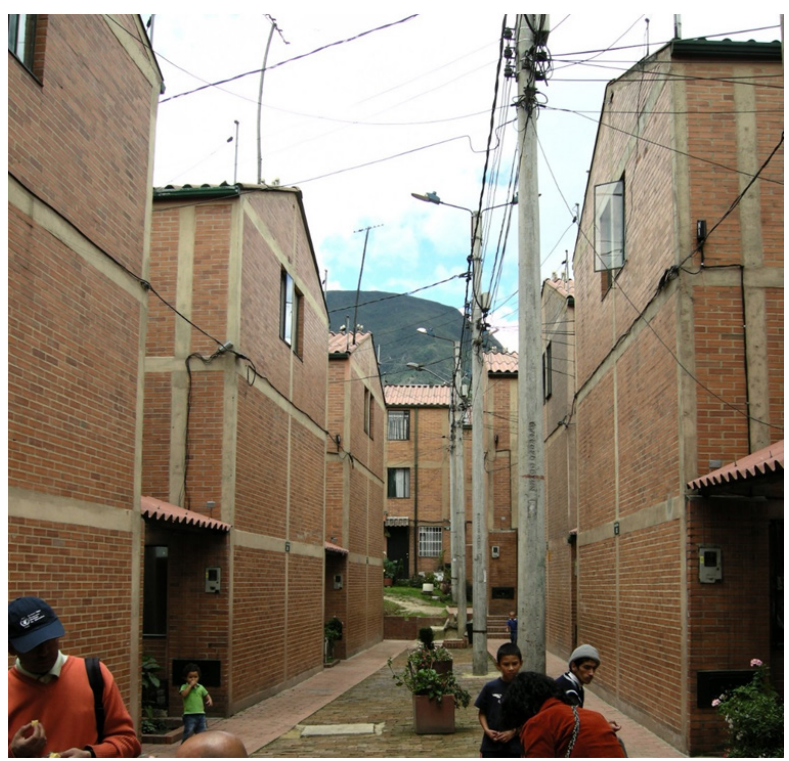

Figura 5. Barrio San Diego, Bogotá. Fuente: Autor (2006) 
En este periodo, se implementó lo dispuesto en el Acuerdo 734 de 1993. En dicha norma se establecen las dimensiones mínimas para los lotes destinados a vivienda social, con los siguientes parámetros; para construcciones unifamiliares se dispone de $12 \mathrm{~m}$ de fondo por $3 \mathrm{~m}$ de frente; para construcciones bifamiliares se establece un fondo de $11 \mathrm{~m}$ y un frente de $6 \mathrm{~m}$; para construcciones trifamiliares, un fondo de $12 \mathrm{~m}$ y un frente de $7 \mathrm{~m}$; y, para construcciones multifamilares, se determina un fondo de $15 \mathrm{~m}$ y un frente de $10 \mathrm{~m}$. Además, añade que cuando se entreguen unidades construidas, las dimensiones de los lotes serán libres.

Así se determinan las siguientes áreas y frentes mínimos de los lotes: para vivienda unifamiliar un área de $60 \mathrm{~m} 2$; para vivienda bifamiliar y multifamiliar $65 \mathrm{~m} 2$; y para vivienda multifamiliar $140 \mathrm{~m} 2$. En esta fase se puede citar, como ejemplo, el barrio San Diego en Bosa, que tiene su origen en 1991. Las viviendas cuentan con un área promedio de $51 \mathrm{~m} 2$ y los lotes $76,8 \mathrm{~m} 2$.

\section{Fase de precaria calidad habitacional en el momento actual.} Decreto 2060 de 2004

Después de la liquidación del Inurbe, se creó el Fondo Nacional de la Vivienda-Fonvivienda, mediante el Decreto 555 de 2003, en el cual también se hace un replanteamiento a la asignación de subsidios, pues solo se otorgarán a las personas que estén dentro de un sistema de ahorros. Además, se reduce el monto del valor promedio de subsidio. Las medidas anteriores fueron tomadas, por un lado, con el fin de que el subsidio se pudiese hacer efectivo en una vivienda (cosa que no estaba sucediendo pues, por la falta de crédito, las familias no podían emplearlo y se quedaba en la asignación), y, por el otro, para que más familias pudieran obtenerlo. Estas medidas han hecho que las familias que no tengan acceso a crédito, por consecuencia, estén imposibilitadas al acceso a una vivienda en condiciones dignas y adecuadas. Además, la producción de VIS actual está centrada en los subsidios asignados a través de las cajas de compensación, lo que ha presentado un alto descenso del aporte estatal en la producción de vivienda.

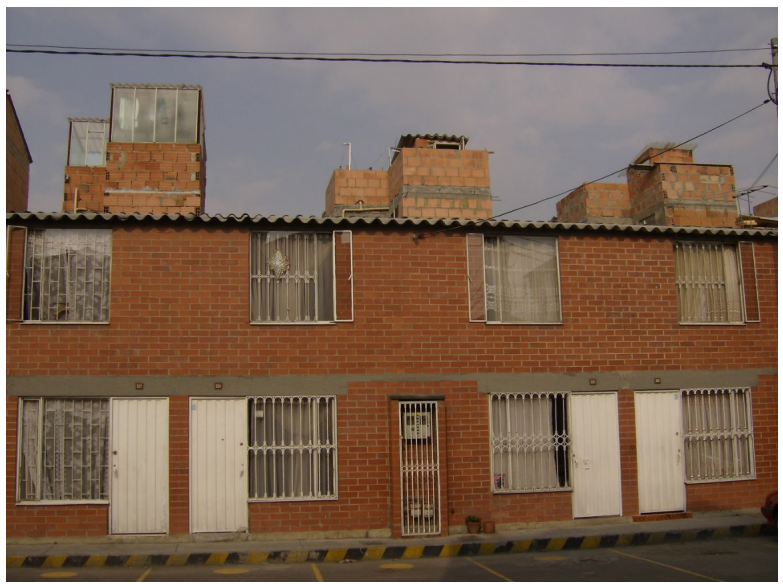

Figura 6. Barrio Ciudadela El Recreo, Bogotá. Fuente: Autor (2008),

Estas políticas se complementan y ejecutan bajo la luz del Decreto 2060 de 2004, a través del cual se regula la dimensión de la vivienda de interés social. En este decreto se establece que el lote para la vivienda unifamiliar mínima es de $35 \mathrm{~m} 2$, que se reparte así: frente mínimo 3,5 m2, y $2 \mathrm{~m} 2$ de aislamiento posterior; para vivienda bifamiliar el lote es de $70 \mathrm{~m} 2$, con un frente mínimo de $7 \mathrm{~m} 2$, y con el aislamiento posterior de $2 \mathrm{~m} 2$; y, por último, para vivienda multifamiliar el lote mínimo es de $120 \mathrm{~m} 2$.

Bajo estos estándares se construye actualmente. Un ejemplo de esto es la vivienda que se ofrece en Ciudadela El Recreo, donde las fachadas y el interior de estas viviendas se entregan en ladrillo a la vista. Además, están dotadas de algunos equipamientos tales como el mesón básico de la cocina, un baño (y sus respectivos aparatos) y el lavadero del patio.

\section{La evolución de la vivienda mínima}

Paulatinamente, esta vivienda se ha reducido en tamaño y en estándares de habitabilidad. En la década de los años sesenta se establecían $120 \mathrm{~m} 2$ para vivienda dirigida a los estratos de menor ingreso; en un segundo momento, en la década de los ochenta, esta área disminuyó a $80 \mathrm{~m} 2$; en el tercer momento, se redujo aún más y se dispusieron $60 \mathrm{~m} 2$; se llega al momento actual con $35 \mathrm{~m} 2$ de vivienda social terminada (ver Figura 7).

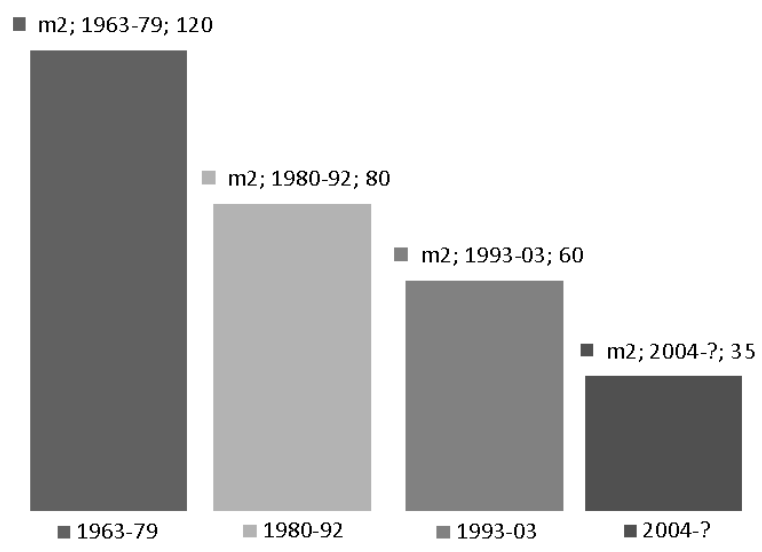

Figura 7. Disminución del área en la vivienda

Fuente: Elaboración propia a partir de información diversa

Por todo lo planteado, se puede concluir que la vivienda en Colombia, a lo largo del siglo XX, ha sufrido diversos cambios. Se ha abordado el tema de la vivienda mínima a través de la adopción de conceptos y tipologías foráneas que, sin embargo, pretendían ser universales. A raíz de esto, se partió de la configuración de la vivienda que planteaba dicho modelo.

En la segunda etapa se plantearon las condiciones físicas y económicas para proyectar un tipo de vivienda de calidad, siguiendo los parámetros modernos, se avanza en las tipologías y se acentúan el patio y el perfil de la vivienda. A partir de la década de los setenta, a raíz de los privilegios que se le dan a las Cajas de Ahorro y Vivienda y al sector privado, se empieza a observar un debilitamiento de esas condiciones $y$, por lo tanto, de la vivienda. Desde este momento, en adelante, se observa un recorte paulatino al lote básico y la vivienda termina convirtiéndose en una mercancía.

\section{Consideraciones finales}

Hasta aquí, se puede concluir que la vivienda mínima no se ha planteado en términos cualitativos. En los orígenes de esta idea, en el seno del movimiento moderno, se planteó como una 
máquina para vivir que sólo variaba de tamaño y en las cuales los planteamientos ligados al reconocimiento de los usuarios no se tradujeron en formas que respondieran a sus necesidades más allá de la muy mencionada higiene.

La vivienda siguió enarbolando estos parámetros modernos y la solución fue producir unidades habitacionales de forma rápida y a bajo costo con el fin de satisfacer la demanda creciente trayendo como consecuencia la extrema estandarización de sus atributos formales y la reducción del significado que puede representar para quien habita en ella.

La idea de la vivienda mínima de la posguerra europea coincidió con los cambios sociales y espaciales que empezaron a sufrir las ciudades latinoamericanas. Por ese motivo, el modelo se convierte en un paradigma.
La tendencia de la política adoptada a lo largo del siglo XX fue la de privatizar. Se puede diferenciar una clara ruptura en los años 80, que se consolida en 1991. En ese punto, el Estado adopta el papel de regulador, abandonando su anterior rol de Estado de Bienestar. Antes de los años 80, la vivienda y el urbanismo dirigido a sectores de bajos ingresos tenía como objetivo el mejoramiento, tanto cuantitativo como cualitativo. Esto se puede ver claramente en los proyectos desarrollados por el ICT.

Desde el Decreto 2060, se han adoptado cambios en las leyes sin ningún tipo de impacto sustancial o cambio de paradigma. El sistema de provisión de vivienda a los sectores de bajos ingresos es, en esencia, el mismo: un sistema basado en el mercado, desarrollado por empresas privadas y regulado por el Estado.

\section{Referencias}

Acevedo, Luis Fernando (2006). Las industrias en el proceso de expansión de Bogotá hacia el occidente. Tesis de maestría de Urbanismo, Facultad de Artes, Universidad Nacional de Colombia, Bogotá.

Aymonino, Carlo (1973). La vivienda racional: ponencias de los congresos CIAM 1929-1930. Barcelona: Editorial Gustavo Gili.

Arango, Gilberto (2001). La vivienda en Colombia en el cambio de siglo, herencias y retos. Trayectorias urbanas en la modernización del Estado en Colombia. Medellín: Editorial Tercer Mundo.

BANCO DE LA REPÚBLICA 2017. El UPAC y la UVR. Bogotá. Recuperado de http://www.banrepcultural.org

CONGRESOS INTERNACIONALES DE ARQUITECTURA MODERNA, CIAM (1954). La carta de Atenas. Buenos Aires: Editorial Contémpora.

Giraldo, Fabio (1994). "Los ciclos de la edificación en Colombia 1950-1994". En: Revista Camacol, No.60, Bogotá.

Pelli, Víctor Saúl (2006). Habitar, participar, pertenecer. Acceder a la vivienda - incluirse en la sociedad. Buenos Aires: Editorial Nobuko.

Rojas, Diana Marcela (2010). La Alianza para el Progreso en Colombia. Análisis Político 23 (70), 91-124.

Roth, André-Noel (2002). Políticas públicas: formulación, implementación y evaluación. Bogotá: Aurora.

Saldarriaga R., Alberto (1996). Estado, ciudad y vivienda: urbanismo y arquitectura de la vivienda estatal, 1918-1990. Bogotá: Inurbe.

Tarchópulos, Doris \& Ceballos, Olga (2003). Calidad de la vivienda dirigida a los sectores de bajos ingresos en Bogotá. Bogotá: CEJA

Notas al final

$1 \quad$ La idea planteada por Le Corbusier consiste en entender la vivienda como una célula conectada con los equipamientos y servicios necesarios para la población, una vivienda complementada con servicios médicos, educación, deporte, zonas verdes, recreación, etc.

2 El concepto de máquina de habitar fue propuesto por Le Corbusier en el IV CIAM, 1933.

3 El Decreto 1579 de 1942 crea la sección de vivienda urbana dentro del ICT. Esta sección asumió funciones similares a las del BCH en cuanto a hacer préstamos a municipios o directamente a los obreros o hacer programas de vivienda. También se otorgó al ICT la facultad de construir "barrios populares modelos" para vender a obreros.

$4 \quad$ La formulación y desarrollo de la APP se conjugaron varios elementos entre los que se destacan el legado de la administración Eisenhower, la influencia de los teóricos de la modernización, y las ideas y energía del gobierno de Kennedy. (Rojas, 2010) 